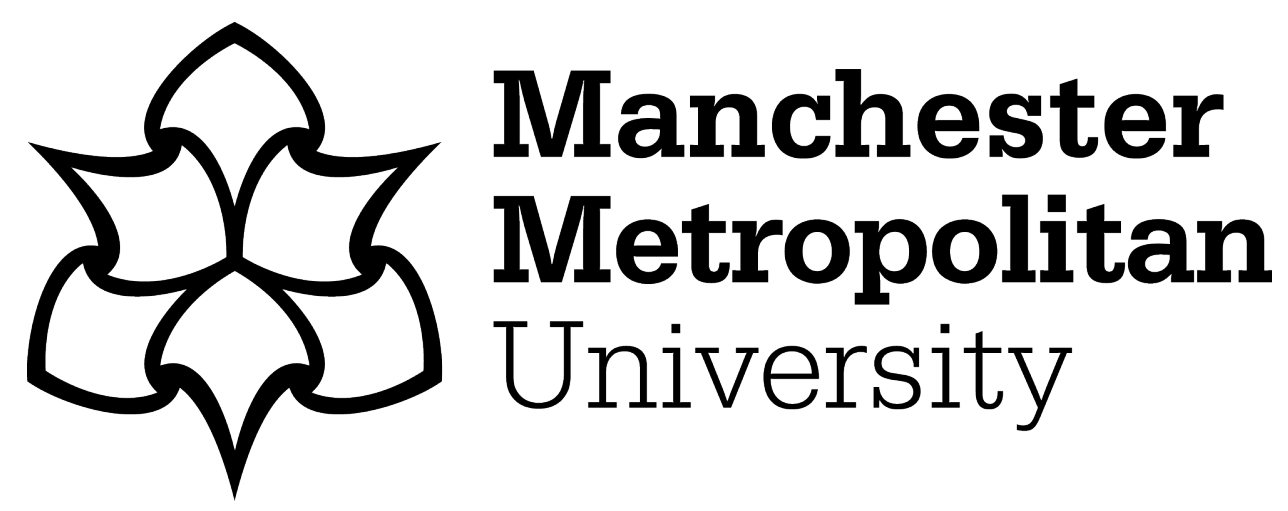

Stanley, CR, Liddiard Williams, H and Preziosi, RF (2018) Female clustering in cockroach aggregations-A case of social niche construction? Ethology, 124 (10). pp. 706-718. ISSN 0179-1613

Downloaded from: https://e-space.mmu.ac.uk/621500/

Version: Accepted Version

Publisher: Wiley

DOI: https://doi.org/10.1111/eth.12799

Please cite the published version 


\title{
Female clustering in cockroach aggregations-A case of social niche construction?
}

\author{
Christina R. Stanley ${ }^{1,2}$ (D) | Huw Liddiard Williams ${ }^{1}$ | Richard F. Preziosi ${ }^{2,3}$
}

${ }^{1}$ Department of Biological Sciences, University of Chester, Chester, UK

${ }^{2}$ Faculty of Life Sciences, University of Manchester, Manchester, UK

${ }^{3}$ School of Science and the Environment, Manchester Metropolitan University, Manchester, UK

\section{Correspondence}

Christina R. Stanley, Department of Biological Sciences, University of Chester, Chester, UK.

Email: c.stanley@cantab.net

Editor: J. Wright

\begin{abstract}
Individuals in groups can suffer costs through interactions with adversarial or unknown conspecifics. Social niche construction allows individuals to buffer such potential costs by only engaging in preferred associations. This may be particularly beneficial in insect aggregations, which are often large and highly fluid. However, little is known regarding the structuring of such aggregations. Here we use social network analyses to test for fine-scale social structure in resting aggregations of the sub-social cockroach Diploptera punctata and to explore the social pressures that contribute towards such structure. We showed that females were significantly more gregarious than males and formed the core of the proximity network, thus demonstrating a higher level of social integration. This fine-scale structure is likely to result from females displacing males; females initiated most displacements whilst males received the majority. We explain this behaviour in terms of social niche construction by showing that females received significantly fewer approaches and investigations at more female-biased local sex ratios. We therefore suggest that female social clustering occurs in this, and presumably other, species to reduce potential costs associated with male harassment. This demonstrates how social niche construction can lead to higher level social structure; we suggest this approach could be used across a range of species in order to improve our understanding of the evolution of sociality.
\end{abstract}

\section{1 | INTRODUCTION}

The social structure of insect groups spans a wide spectrum, from temporary, loose feeding aggregations with no obvious underlying structure to highly stable eusocial colonies exhibiting multiple levels of organisation (Fewell, 2003) and information transfer rivalling that of man-made networks (Charbonneau, Blonder, \& Dornhaus, 2013). Many species cope well with life in the laboratory and have simple husbandry requirements, meaning they are well suited to behavioural experiments (Kralj-Fiser \& Schuett, 2014). Despite this, little is known regarding the level of social structuring in insect aggregations, aside from that in aggregations formed exclusively for

This work was carried out at the University of Manchester and the University of Chester. The current affiliations for Christina Stanley and Richard Preziosi are University of Chester and Manchester Metropolitan University, respectively. mating. Identification of such structure, as well as the behavioural algorithms followed by individual animals leading to it, could be key to our understanding of collective behaviour in animals (Sumpter, 2006). Indeed, much of the research to date on insect aggregations concerns their proximate causes and emergent properties. Large aggregations can occur due to the attraction of individuals to a resource (Wilson \& Richards, 2000) or due to mutual attraction between group members (Jeanson et al., 2005). Properties such as information centralisation and collective decision-making may then emerge which are not possible at the individual level (Parrish \& Edelstein-Keshet, 1999). Mating strategies could also play a key role in the structuring of insect aggregations (Fisher, Rodríguez-Muñoz, \& Tregenza, 2016a; Inghilesi, Mazza, Cervo, \& Cini, 2015; Muniz, Guimarães, Buzatto, \& Machado, 2014). However, the fine-scale social structure of non-eusocial aggregations, and the individual 
decisions leading to this are largely unexplored; this is especially true for cockroaches (Blattodea).

Despite intensive study of cockroach species in the fields of molecular biology, neuroscience and physiology, this taxon remains massively under-represented in the behavioural sciences (Lihoreau, Costa, \& Rivault, 2012) and cockroach social behaviour has so far rarely been studied (Costa, 2006). Relatively sophisticated communication (using cuticular hydrocarbons secreted on the body surface or deposited on the substrate) and emergent forms of cooperation have been identified in cockroaches (Lihoreau et al., 2012). Our aim here is therefore to explore fine-scale social structure in resting cockroach aggregations and to provide potential explanations for this structure in terms of the individual decisions that lead to its formation.

Fine-scale social structure in animals is the result of individual association preferences; social niche construction (where individuals influence the composition and dynamics of their social environments, Saltz, Geiger, Anderson, Johnson, \& Marren, 2016) could provide an ultimate explanation for these individual differences in behaviour (Bergmüller \& Taborsky, 2010). Association with particular conspecifics can act as a buffer to fluctuations in the social environment, such as interactions with adversaries and the potential costs of novel interactions. For animals living in groups whose membership is constantly changing such as temporary cockroach aggregations, there is high variability in the social environment. This can incur costs, especially in terms of aggression and harassment (Pilastro, Benetton, \& Bisazza, 2003). Social niches can therefore be constructed by individuals to limit the variability they experience, thereby maximising their inclusive fitness (Saltz et al., 2016). There are numerous examples of social niche construction across the animal kingdom; female capuchins Cebus capuchinus form coalitions and as a result sustain their social ranks in a changing social environment (Silk, 2007), female brown-headed cowbirds Molothrus ater consistently associate with other females across changing group composition and thereby benefit from social information (Kohn, King, Scherschel, \& West, 2011) and in the Trinidadian guppy Poecilia reticulata, non-receptive females that associate with receptive females avoid male harassment (Brask, Croft, Thompson, Dabelsteen, \& Darden, 2012). Social niche construction can have clear inclusive fitness benefits; the genotypes of Drosophila melanogaster with the highest mating success were found to be those whose social niche construction behaviour generated the most favourable social environment for their particular mating strategy (Saltz \& Foley, 2011). The identification of social niche construction in insect aggregations can therefore be used to elucidate the social pressures experienced by individuals that lead to certain association preferences and higher level social structure.

There are often benefits for animals in associating with those sharing certain characteristics; this so-called homophily of associations is widespread in nature (e.g., sex- and age-based homophily have been demonstrated in zebra Equus grevyi, Sundaresan, Fischhoff, Dushoff, \& Rubenstein, 2007; and dolphins Tursiops sp., Lusseau \& Newman, 2004) and it can evolve under a variety of conditions (Fu, Nowak, Christakis, \& Fowler, 2012). For cockroaches, simple decisions regarding choice of associates are likely to be made based on shared kinship levels and sex as both can be assessed relatively easily. Sex recognition in cockroaches occurs through contact chemoreception via the antennae (Fukui \& Takahashi, 1980); a contact pheromone is present in the cuticular hydrocarbons which allows very quick discrimination between males and females (Lihoreau \& Rivault, 2009; Seelinger \& Schuderer, 1985). In a similar way, cockroaches have also been shown to possess the ability to recognise close kin via cuticular hydrocarbons (Lihoreau \& Rivault, 2009). Preferential association with close kin has already been demonstrated in the German cockroach Blatella germanica and could lead to subgroups of kin in large resting aggregations (Lihoreau \& Rivault, 2009). Indeed, another study on this species revealed a pattern of decreasing relatedness by distance due to patterns of dispersal and isolation on a fine enough scale to indicate active individual choices (Crissman et al., 2010). However, cockroach aggregations often contain many strains (Ame, Rivault, \& Deneubourg, 2004) and genetically distinct individuals have been found to enter without eliciting either aggression or rejection (Sempo, Canonge, Detrain, \& Deneubourg, 2009). Sex is another potential factor that could influence cockroach association preferences since many cockroach species show distinct sexual dimorphism (Costa, 2006) and benefits of female social clustering have been demonstrated in other species; sexbased association is, however, yet to be explored in cockroach aggregations.

Sex-based associations are common across the animal kingdom; in terms of social niche construction, females in particular often choose to cluster with each other in order to minimise harassment from males. Sexual harassment from multiple males can considerably reduce female fitness (Clutton-Brock \& Parker, 1995). The costs of mating, particularly to females, have been well-known for some time (Daly, 1978), especially where sexual coercion is achieved by force (Smuts \& Smuts, 1993) and results in increased mortality rates (Reale, Bousses, \& Chapuis, 1996). Whilst there are clear benefits to polyandry for female insects in terms of increased lifetime offspring production (Arnqvist \& Nilsson, 2000), repeated attempts at courtship can also be significantly costly to females in terms of a loss in feeding time, increased energy expenditure and an increased risk of predation (Arnqvist \& Nilsson, 2000; Clutton-Brock \& Parker, 1995; Krupa \& Sih, 1993). Female social clustering can therefore be beneficial in terms of limiting male harassment and improving reproductive success (Linklater, Cameron, Minot, \& Stafford, 1999). We therefore expect there to be some degree of female social clustering in cockroach aggregations that could be explained by social niche construction.

To test this prediction, we observed association choices and behaviour in a laboratory colony of the species Diploptera punctata. Also known as the Pacific beetle roach, D. punctata is a species of cockroach that is widely used as a model system for the study of the endocrinology of arthropod reproduction and development 
(Marchal, Hult, Huang, Stay, \& Tobe, 2013). It has also been used to study group effects (Holbrook \& Schal, 1998) and phenotypic plasticity (Holbrook \& Schal, 2004), as well as the development of personality (Stanley, Mettke-Hofmann, \& Preziosi, 2017). Despite this, its social structure has not yet been explored and very little is known regarding its behavioural ecology or life history. Diploptera punctata are difficult to study in their natural habitat as they live mostly in leaf litter (Costa, 2006). There is consequently very little published material on the natural behaviour of this species, although $D$. punctata are known to mate with multiple males in the laboratory, sometimes resulting in mixed paternity broods (Woodhead, 1985). Repeated mating can involve significant costs to females (Lange, Reinhardt, Michiels, \& Anthes, 2013; Rowe, 1994); we might therefore expect some level of social niche construction to occur in D. punctata as a way of minimising potentially costly male harassment.

We carried out a combination of experiments to test two main hypotheses: that there will be some degree of fine-scale social structure present in resting cockroach aggregations, specifically in terms of female social clustering, and that social pressures experienced by females will provide an explanation for this observed structure. In order to test for significant fine-scale social structure, we observed four mixed-sex cockroach groups in purposebuilt arenas and built proximity networks based on individuals' nearest neighbours to examine association choices and fine-scale social structure using social network methods. Social network analysis provides a powerful toolkit for the exploration of animal social preferences (Krause, Croft, \& James, 2007; Kurvers, Krause, Croft, Wilson, \& Wolf, 2014; Sih, Hanser, \& McHugh, 2009; Wey, Blumstein, Shen, \& Jordan, 2008). Our understanding of colony organisation and stability in the eusocial insects has been significantly improved by the application of network analyses (e.g., Charbonneau et al., 2013; Fewell, 2003; Jeanson, 2012; Naug, 2009). However, other insect species, many of which show levels of sociality approaching those of the eusocial insects (see Costa, 2006), have been almost entirely overlooked (but see Formica et al., 2012; and Fisher, Rodríguez-Muñoz, \& Tregenza, 2016b, for rare examples). In our study, we therefore chose social network analysis as a tool to improve our understanding of insect social associations. We used two different approaches to test for the presence of social pressures that could explain these association choices. Firstly, we examined approach and displacement networks built for two of the resting aggregations in the aforementioned arenas in order to test for sex differences in behavioural interactions given and received that could lead to finescale social structure. Secondly, we observed female behaviour in artificially constructed social groups with varying sex ratios in order to quantify social pressures that could explain the observed association choices. If a female experiences a more beneficial social environment (for example, where male approaches are less frequent) where the female:male sex ratio is higher, it would pay for females to associate together and thus construct a more favourable social niche.

\section{2 | MATERIALS AND METHODS}

\section{1 | Study population}

Study individuals were taken from a stock population of D. punctata cockroaches that had been reared in laboratory conditions for a minimum of 10 generations at the University of Manchester. These were kept in an incubator at $24.5^{\circ} \mathrm{C}$ with a $12: 12$ light:dark cycle in plastic tanks approximately 33 by 26 by $19 \mathrm{~cm}$. Holes in the lids provided ventilation. These cockroaches were allowed to feed ad libitum on Lidl's "Orlando complete" dog biscuits and were given a constant supply of fresh water.

\section{2 | Experiment 1: Social network study}

\subsection{1 | Protocol}

In order to investigate four social groups, four $21 \times 30 \mathrm{~cm}$ arenas were constructed using sheets of transparent Perspex held $0.5 \mathrm{~cm}$ apart by "walls" of draught-insulation foam. Cockroaches could move around freely, with there being sufficient room for two or more isolated aggregations to form, but adults were not able to either climb on top of other individuals or to walk on the ceiling. Two-dimensional habitats such as this would be used by cockroach aggregations in their natural environment, for example under logs. Ventilation gaps approximately $10 \mathrm{~cm}$ wide were made using nylon mesh fabric at opposite ends of the arena and water was provided by a soaked cotton wool ball. "Aquarian" fish flakes were provided in a shallow plastic dish. The arenas were maintained in the same conditions as the original colony. CCTV video cameras (CCTV42, Buckinghamshire) were mounted $50 \mathrm{~cm}$ above the arenas and filmed the entire arena at a speed of two frames per second. Images were recorded on a System 2.1 Digital Video Recorder (CCTV42) and viewed using SuperPlay software 1.2.1 (AAT Holdings, Warsaw, Poland).

Five female and five male adults were randomly selected from the colony to inhabit each arena (40 individuals in total). Since little is known about this species' behavioural ecology, this was deemed to be a sufficient size as natural aggregations are frequently limited by the holding capacity of the shelter (Appel \& Rust, 1985), meaning aggregations of this size are likely to occur in the natural environment. By including five individuals of each sex, this allowed all individuals a sufficient choice of associates both within and between sexes. The sex ratio of 1:1 was consistent with that found in wild colonies (Lihoreau et al., 2012). Individuals were marked by attaching a 3-mm diameter coloured paper disk to the pronotum using glue (Bostik Glu $\&$ Fix). Individuals were also marked with either a blue or red nail varnish dot, or no dot, on their abdomen. The combination of coloured disk and dot was unique for each individual in each arena. Individuals were allowed $24 \mathrm{hr}$ to habituate to their new markings (all adults were housed together during this period) before being introduced to the arena, along with five nymphs per arena randomly selected from the stock population (currently between first and third instar stages) in order to mimic the age structure of natural colonies (Lihoreau 
et al., 2012). Nymphs were not individually marked (as they would lose markings when they moulted) and were therefore omitted from all analyses. Similar markings were used for a previous study (Stanley et al., 2017) and were not found to have an observable effect on behaviour in this species.

The social arenas were set up in September 2014 at the University of Manchester and were constantly filmed for 21 days. Diploptera punctata does not show a distinct mating season in laboratory conditions, and females are continually observed to mate and produce young throughout the year (personal observation, CS). Individuals could only be distinguished from each other during daylight hours; although this species is most active at night, D. punctata are also known to forage during the day (Bell, Roth, \& Nalepa, 2007) and there was sufficient activity during this time for multiple changes in individual positions. Footage was downloaded and transcribed at the end of the period of filming by CS.

\subsection{2 | Social networks}

Dyadic associations were recorded at four hourly intervals during the light hours (09.00 to 21.00) for 21 days, giving a total of 85 observations for each individual in each of the four arenas. This sampling regime was similar to that used for a comparable study on beetles (Formica et al., 2012). To achieve this, we froze the video footage at four hourly intervals and recorded the identity of the nearest neighbour (or multiple neighbours, if equidistant) for each individual in each arena at this time point. The simple ratio index (Cairns \& Schwager, 1987) was then used to calculate an association index for each potential dyad; this results in a score varying between zero (for no association being recorded) and one (for two individuals that were constantly associated). This was calculated using the following formula:

$$
\frac{x}{x+y}
$$

where $x$ is the number of times individual $B$ was recorded as nearest neighbour to individual $A$ and $y$ is the number of observations where individual $B$ was not recorded as nearest neighbour to individual A. This resulted in four asymmetrical association matrices. Proximity networks, showing the strength of the association between individuals based on the frequency of them being nearest neighbours, were then built from these matrices using UCINET 6 (Borgatti, Everett, \& Freeman, 2002); proximity networks have previously been used as a valid proxy for behavioural interactions in insects (Jeanson, 2012) and are especially useful where little is known of a species' social behaviour. Networks for each of the four arenas were visualised using NetDraw (Borgatti, 2002).

Behavioural interactions were then sampled from footage filmed in arenas one and two. Two behaviours were scored: approach and displacement. Approach behaviour was defined as one individual approaching another to within one body length without the other individual immediately moving away, whilst displacement was defined as one individual moving away when another individual approached it to within one body length. All incidences of these behaviours were recorded using all occurrence sampling (Altmann, 1974) during ten 30-min sampling periods spread equally throughout the daylight hours, over a randomly selected 5 -day period. This gave a total of 300 min of behavioural observations for each of the two arenas. Since strong sex differences in behaviour were evident during this period and sex differences in individual centrality levels were found to be consistent across the two arenas, this level of sampling was deemed sufficient; there was insufficient time and manpower available to sample all four arenas.

The total number of times each individual displaced every other individual was recorded in a displacement matrix and resulted in a displacement network, whilst the total number of times each individual approached every other individual formed an approach matrix and network. The total number of approaches/displacements in every cell was weighted by dividing by $(m+1)$, where $m$ was the maximum cell value in the matrix. This gave weighted association indices varying between zero and one, with higher numbers representing a larger proportion of events.

\section{3 | Experiment 2: Sex ratio manipulation}

This experiment was carried out between November 2016 and January 2017 by HLW at the University of Chester to explore the social pressures experienced by females at varying sex ratios. An observation enclosure measuring 16 by 10.5 by $8 \mathrm{~cm}$, made from opaque plastic (to eliminate exposure to visual external stimuli), was set up with Vaseline petroleum jelly applied approximately $5 \mathrm{~cm}$ above the base to prevent cockroaches from climbing the container walls. Between trials, this was cleaned using antibacterial wash to remove any cuticular hydrocarbons or other matter deposited by previous individuals. The temperature was maintained at $22^{\circ} \mathrm{C}$ throughout observations; this differed from the temperature of $24.5^{\circ} \mathrm{C}$ used for the experiments at the University of Manchester, but was constant across treatments, allowing unbiased comparisons across sex ratios.

Males' and females' abdomens were marked with blue or red nail varnish to distinguish between the sexes. They were allowed a minimum of $24 \mathrm{hr}$ to habituate to these markings prior to being used in a trial. Individuals were selected at random from the mass colony (a satellite colony to that used at the University of Manchester) and allowed $5 \mathrm{~min}$ to habituate to the enclosure once introduced. They were then filmed using a camera (GoPro Hero 4) mounted $13 \mathrm{~cm}$ vertically from the base of the enclosure, with footage being recorded at a rate of two frames per second for a period of $30 \mathrm{~min}$.

A total of six individuals were introduced to the enclosure in each trial at a sex ratio of either $5: 1,4: 2,3: 3,2: 4$ or $1: 5$ males to females. Each sex ratio treatment was used 10 times, giving a total of 50 trials. Group composition (in terms of the individuals present) was changed between trials within each day, but as all individuals were then returned to the main colony at the end of the day's testing, the same group composition could theoretically have been repeated on a different day. 


\begin{tabular}{|l|l|l|l}
\hline Network statistic & Definition \\
\hline Strength centrality & $\begin{array}{c}\text { The sum of weights on edges originating from a node (out-strength) and } \\
\text { entering a node (in-strength). At a network level, mean strength } \\
\text { centrality gives a measure of the average gregariousness across all } \\
\text { individuals in the network }\end{array}$ \\
\begin{tabular}{|l} 
The shortest distance between two nodes, accounting for the edge \\
weights included within this path. The reciprocal of edge weights is \\
used as a measure of "cost", so that edges with higher weights have \\
lower cost and therefore less resistance to information flow. At a \\
network level, mean path length gives a measure of how well \\
connected individuals are to each other within the network
\end{tabular} \\
$\begin{array}{l}\text { This is a measure of how well the associates of an individual are } \\
\text { themselves associated. The mean therefore gives a measure of how } \\
\text { frequently clusters of well-connected individuals occur in the network }\end{array}$ \\
\hline $\begin{array}{l}\text { This is a measure of indirect connectedness, i.e., how easily information } \\
\text { from one individual can reach others in the network via indirect routes. } \\
\text { It is defined as the sum of the products of all pairs of association } \\
\text { indices which link two nodes through a third individual }\end{array}$ \\
\hline Reach
\end{tabular}

TABLE 1 Definitions of weighted network measures calculated (Wasserman \& Faust, 1994)
Footage was later viewed and transcribed by HLW. Focal all occurrence sampling (Altmann, 1974) was used to record the rates of displacement (when an individual changes their position within 2-s of being approached to within antennal range by any other individual), approach (when an individual is approached to within antennal range by any other individual but does not change its position within 2-s) and investigation (as for approach, but also includes sustained antennal contact with the focal individual's body for more than 2-s by the approaching individual) experienced by one female. The definitions for approach and displacement differed from those used in the first experiment as these were defined in terms of movement within another individual's antennal range instead of body length; however, these distances are very similar in practice. Investigation was included here (but not in the social networks experiment) so that we could investigate the females' social environment in more detail here. One focal female was selected at random from those females present in each trial (although there was no choice of focal in the 5:1 treatment) and was observed for the full 30-min observation period, following the 5-min habituation period. All instances of displacement, approach and investigation received by the focal female were recorded and later converted to a rate per minute for these behavioural events. This allowed quantification of the females' social environment at differing sex ratios.

\section{4 | Statistical analyses}

To test for structural consistency across networks, the following network measures were calculated for each weighted proximity network: path length (maximum and mean), mean clustering coefficient, mean reach and mean in- and out-strength centrality (Opsahl \& Panzarasa, 2009; Wasserman \& Faust, 1994), see Table 1 for definitions and interpretations of these measures). Path length was calculated in the package tnet (Opsahl, 2009) in the R environment (R Development Core Team, 2013) whilst strength, reach and clustering coefficients were calculated using SOCPROG 2.5 (Whitehead, 2009).

\subsection{1 | Exploration of fine-scale social structure}

To determine whether females and males differed in terms of gregariousness, both in-strength and out-strength centrality (see Table 1 for definitions) were calculated for each individual within each proximity network. For this proximity network, out-strength centrality measures how frequently an individual is the nearest neighbour of other individuals, and in-strength centrality gives a measure of the strength of association with other individuals (with a larger value indicating an individual has a larger number of neighbours or more frequent associations with these individuals). Individuals with more central network positions are thought to have more influence in a network (Croft, James, \& Krause, 2008; Wasserman \& Faust, 1994). Permuted $t$-tests using 10,000 permutations were carried out in UCINET to test for a significant effect of sex on centrality values (pooled across all four networks). This test takes the two categories (male and female) and randomly assigns the measures (individual centrality measures across all arenas) to each category, keeping the total number in each category the same to generate a sampling distribution of the difference between the categories' means (Hanneman \& Riddle, 2005). Pooling all individuals' absolute centrality values was justifiable in this case since all four networks had the same number of actors (Wasserman \& Faust, 1994).

The level of social integration of individuals within the network was also calculated to explore whether this differed between sexes. Individuals can be assigned either to a graph's core (a partially complete subgraph, i.e., a highly connected group) or its periphery (a collection of actors which do not interact greatly with each other) using a block modelling approach to classify their contribution to the network in a binary fashion (Borgatti \& Everett, 1999; Everett $\&$ Borgatti, 2005). Individuals that form the core of the network all have high centrality levels; however, the reverse is not always true, 
as an individual can be highly central and yet not form part of a core highly connected sub-group (Borgatti \& Everett, 1999). Since not all networks have a distinct "core" and a distinct "periphery", "coreness" values can also be calculated which quantify continuously the extent to which a node belongs to the core group (Borgatti \& Everett, 1999; Everett \& Borgatti, 2005). If coreness values show little difference between individuals, the network to which they belong does not have a discrete highly connected core (Carrington, Scott, $\&$ Wasserman, 2005). The core/periphery command in UCINET was used to calculate both these measures (Everett \& Borgatti, 2005) to determine individuals' contributions to the core of the network. A permuted t-test (with 10,000 permutations) was then carried out in UCINET to determine whether there was a significant influence of sex on coreness values. Values are reported as $\bar{X} \pm S D$. For analyses in this section, the sample size was considered to be the number of replicate arenas as it was the network structure that was being examined.

\subsection{2 | Exploration of social pressures explaining observed fine-scale structure}

To determine whether behavioural interactions have a significant influence on the fine-scale social structure elucidated in the proximity network, the Quadratic Assignment Procedure (QAP) with 10,000 permutations was used to determine whether there were any significant correlations between weighted proximity networks and their associated approach and displacement networks (for the two arenas from which social interactions were sampled) in UCINET. This procedure carries out linked permutations of rows and columns of the observed matrix in order to generate multiple permuted matrices, which are then correlated with the dependent matrix in turn to calculate the probability that an observed correlation is significantly higher than expected (Borgatti, Everett, \& Freeman, 2006; Krackhardt, 1988); it is therefore functionally equivalent to the Mantel test (Legendre \& Fortin, 2010). If either behavioural network correlated with the proximity network, either negatively or positively, we can infer that these behaviours have an influence on individuals' choices of associations.

To explore sex differences in behavioural interactions both initiated and received, in-strength and out-strength centrality measures were calculated for each individual within both the approach and displacement networks for the two arenas sampled. These measure how frequently individuals were approached and displaced (instrength), as well as how frequently they approached and displaced others (out-strength). Permuted t-tests in UCINET were used to test for a significant effect of sex on these centrality values. Individuals were also assigned dominance scores to improve our understanding of each sex's relative influence on social dynamics. To do this, individual David's scores (David, 1988) were calculated from the displacement networks in SOCPROG.

To quantify differences in the social environment experienced by females at different sex ratios, we carried out correlation tests between the rates of each behaviour experienced and the sex ratio, which was treated as a continuous variable for this purpose. Since behavioural frequency data could not be transformed to fit a normal distribution, a Spearman's correlation test was carried out in the $R$ environment to determine if there was a significant correlation between the rates of each of the three behaviours experienced and an increasingly male-biased sex ratio. The sample size for this analysis was taken to be the number of replicate trials for each sex ratio.

\section{5 | Ethical note}

We did not observe any adverse effects from temporarily housing these cockroaches in artificially constructed test arenas. The minimum number of individuals necessary to test the hypotheses was used and all animals were returned to the mass colony following the end of the experiment. Environmental enrichment (cardboard "egg boxes" to provide shelter and a more stimulating environment) was used in the mass colonies.

\section{3 | RESULTS}

The number of data points per arena for proximity networks differed slightly due to poorer quality recording at certain time points (Arena 1: $N=85$; Arena 2: $N=82$; Arena 3: $N=75$; Arena 4: $N=82$ ). One individual in Arena 1 died between days 14 and 15; 54 data points were therefore available for this individual, which was deemed adequate for the analysis so this individual was included, with its weighting being calculated using the total number of possible observations as 54 instead of 85 .

Proximity networks for the four arenas were structurally similar; network-level statistics were of a similar magnitude across all four networks, indicating comparable social structures across the four groups in terms of clustering and the potential for information flow (Figure 1, Table 2). Across both arenas from which social behaviour was sampled, approaches were much more frequent than displacements (approaches: $1.017 \pm 0.62$ events per min, displacements: $0.198 \pm 0.28$ events per min). In the experiment where sex ratio was manipulated, the frequency of displacements received by the focal female in the equal sex ratios treatment was also substantially lower than the frequency of approaches (approaches: $0.250 \pm 0.10$ events per min, displacements: $0.040 \pm 0.03$ events per min).

\section{1 | Exploration of fine-scale social structure}

Females were more gregarious than males; females were assigned significantly higher centrality measures for both weighted instrength (males: $1.331 \pm 0.057$; females: $1.392 \pm 0.097$; permuted t-test: $P=0.022$ ) and out-strength (males: $1.282 \pm 0.172$; females: $1.441 \pm 0.299$; permuted $t$-test: $P=0.049$ ) centrality across proximity networks.

Females also showed a higher level of social integration than males, as highlighted by the core/periphery analysis. Whilst 13 of 20 females were assigned membership to the core of the proximity 

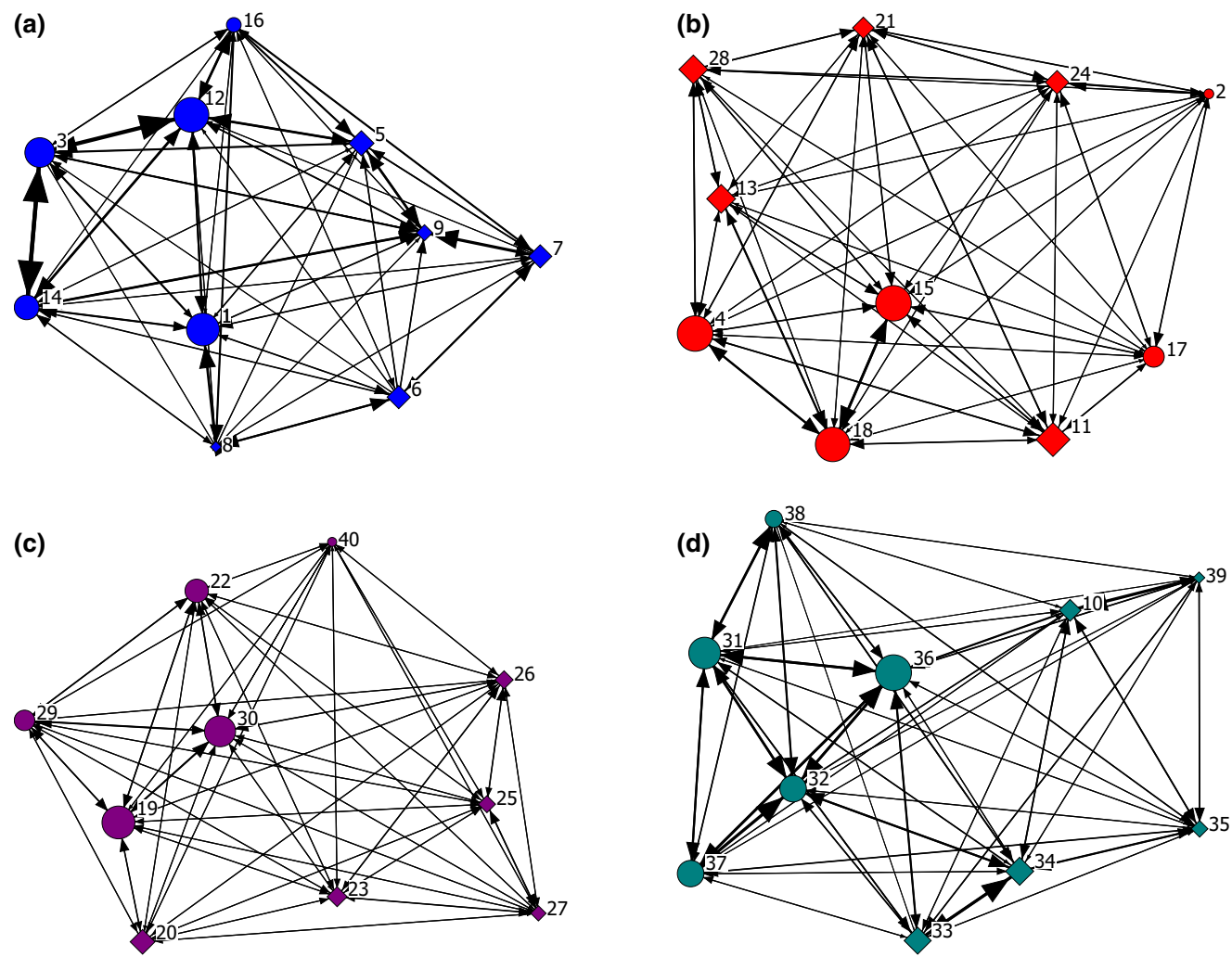

FIGURE 1 Weighted proximity networks for (a) Arena 1, (b) Arena 2, (c) Arena 3 and (d) Arena 4. Thickness of lines indicates frequency that two individuals were recorded as nearest neighbours, with arrow sizes depicting directionality of relationship (with a bigger arrow representing one individual is more frequently the nearest neighbour of the other). Node shapes represent sex (circle = female, diamond $=$ male) and node size increases with out-strength centrality [Colour figure can be viewed at wileyonlinelibrary.com]

\begin{tabular}{|lllll|}
\hline Arena & 1 & 2 & 3 & 4 \\
\hline $\begin{array}{l}\text { Network statistic } \\
\text { Max path length }\end{array}$ & 1.96 & 2.01 & 1.86 & 2.01 \\
\hline Mean path length & $1.08(0.34)$ & $1.10(0.38)$ & $1.09(0.37)$ & $1.09(0.36)$ \\
\hline $\begin{array}{c}\text { Mean clustering } \\
\text { coefficient }\end{array}$ & $0.47(0.01)$ & $0.48(0.02)$ & $0.47(0.02)$ & $0.58(0.02)$ \\
\hline $\begin{array}{l}\text { Mean reach } \\
\text { Mean out-strength } \\
\text { centrality }\end{array}$ & $1.97(0.21)$ & $1.78(0.24)$ & $1.82(0.25)$ & $1.95(0.24)$ \\
\hline
\end{tabular}

TABLE 2 Summary network statistics for each of the four arenas' proximity networks. Standard deviations are given in parentheses where appropriate.

Definitions of measures are given in Methods (Table 1) networks, only one of 20 males was assigned to the core, with the remainder being allocated peripheral status. Continuous coreness values were significantly higher for females $(0.323 \pm 0.013)$ than for males (0.309 \pm 0.020 ; permuted $t$-test: $P=0.015)$.

\section{2 | Exploration of social pressures underlying association choices}

There was no evidence that either behavioural network alone explained the associations identified in the proximity network. There were no significant pairwise correlations between the proximity network and its associated approach or displacement network for either arena tested (QAP tests, Arena 1: approach vs. proximity $r=-0.10$,
$P=0.101$; displacement vs. proximity, $r=-0.02, P=0.400$; approach vs. displacement, $r=-0.15, P=0.139$; Arena 2: approach vs. proximity $r=-0.11, P=0.121$; displacement vs. proximity, $r=0.07$, $P=0.242$; approach vs. displacement, $r=-0.13, P=0.152$ ).

Comparisons of in-strength centrality values revealed that significantly more approaches were received by females than by males (females: $2.26 \pm 0.58$; males: $1.25 \pm 0.36$; permuted t-test: $P<0.001$, Figure 2) across both arenas. Males tended to instigate more approaches, but this difference was not significant at the $\alpha=0.05$ level from comparisons of out-strength centrality measures (females: $1.26 \pm 0.47$; males: $2.25 \pm 1.56$; permuted $t$-test: $P=0.080$ ). In the displacement networks, a comparison of strength centrality values revealed that significantly more displacements were instigated by 
(a)

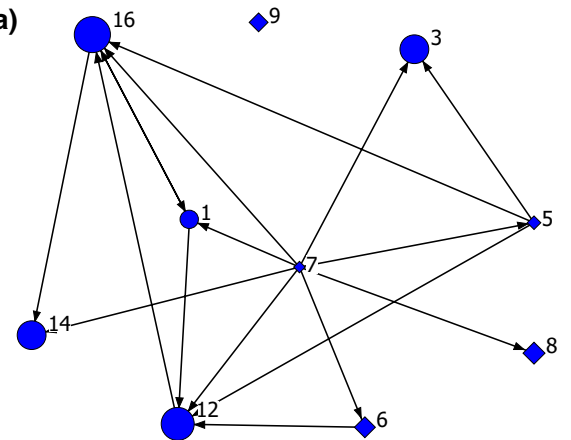

(c)

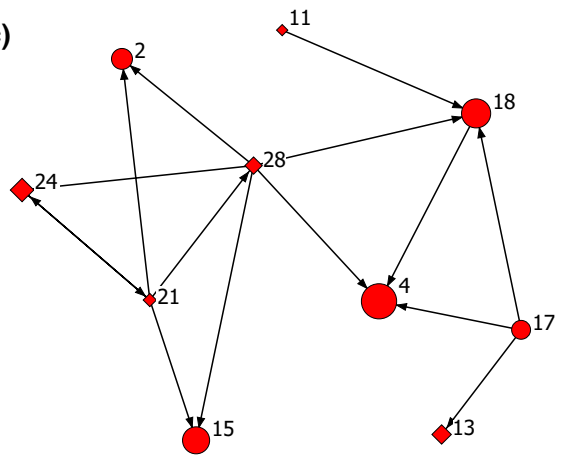

(b)

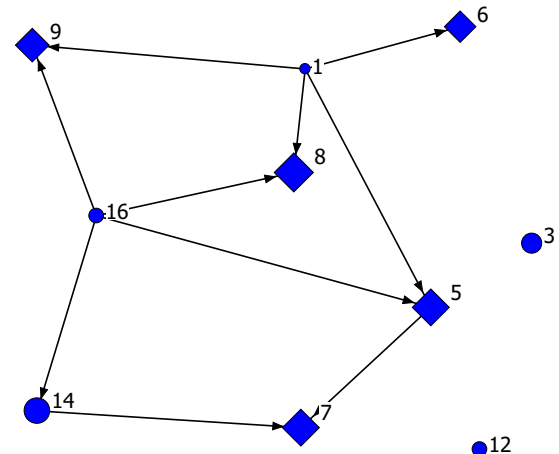

(d)

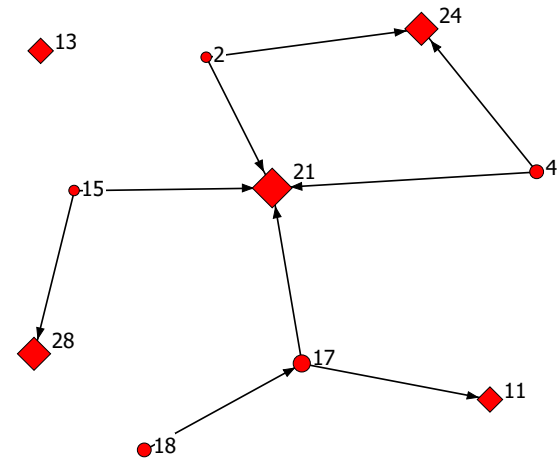

FIGURE 2 Arena 1 (a) approach and (b) displacement networks and Arena 2 (c) approach and (d) displacement networks. These directed binary networks represent the strongest ties occurring in the weighted networks and were created by changing the threshold until around 15 ties remained (threshold used was 0.35 for approaches and 0.2 for displacements). Node shapes represent sex (circle $=$ female, diamond $=$ male). Node size increases with in-strength centrality [Colour figure can be viewed at wileyonlinelibrary.com]

females as shown by differences in mean out-strength centrality values (females: $1.21 \pm 0.70$; males: $0.21 \pm 0.15$; permuted $t$-test: $P<0.001$ ). Significantly more displacements were received by males, as shown by differences in mean in-strength centrality values (females: $0.25 \pm 0.24$; males: $1.17 \pm 0.24$; permuted t-test: $P<0.001$, Figure 2 ). Dominance ranks assigned using David's scores placed all females above all males in both networks (Mean David's scores: females 10.8 \pm 7.4 , males $-10.8 \pm 4.7)$.

The local sex ratio had an impact on females' social environments (Figure 3). In increasingly male-biased sex ratios, females experienced significantly more investigations (Spearman's correlation test: $r_{s}=0.554, n=50, P<0.001$ ) and approaches (Spearman's correlation test: $r_{s}=0.455, n=50, P<0.001$ ), but there was no correlation with the rate of displacements received (Spearman's correlation test: $r_{s}=0.234, n=50, P=0.102$ ).

\section{DISCUSSION}

Female social niche construction has been demonstrated across a range of animal species. Here we used social network analyses to reveal fine-scale social structure in resting aggregations of the cockroach $D$. punctata that can be explained by female social niche construction; we showed that females were significantly more gregarious than males and formed the core of the proximity network, thus showing a higher level of social integration. This fine-scale structure cannot be explained by displacement or approach behaviour alone. Instead, it is likely to result from females displacing males; females were dominant to males, with most displacements being instigated by females and received by males. We explain this behaviour in terms of social niche construction by showing that at more female-biased local sex ratios, females were likely to have a more favourable social environment as they received significantly fewer approaches and investigations. We therefore suggest that female social clustering occurs in this species, as in others, as an attempt to reduce potential costs associated with male harassment.

Fine-scale social structure, specifically in terms of sex differences in gregariousness and social integration, was clearly evident in experimentally constructed cockroach resting aggregations. Four separate groups of D. punctata were studied, all comprising equal male: female sex ratios and group sizes; a 21 day period gave individuals adequate time to frequently change resting positions and to choose associations with either sex. Across all four proximity networks, females were more gregarious than males; both out-degree and in-degree centrality values were significantly higher in females, signifying either a greater number or a higher strength of spatial association with neighbours than was found in males. Females also showed higher levels of social integration than males as they were assigned to all but one of the core network positions in these four networks, demonstrating that it is clusters of females that form the centre of these networks (Borgatti \& Everett, 1999). In addition, coreness values, quantifying the level of an individual's contribution 

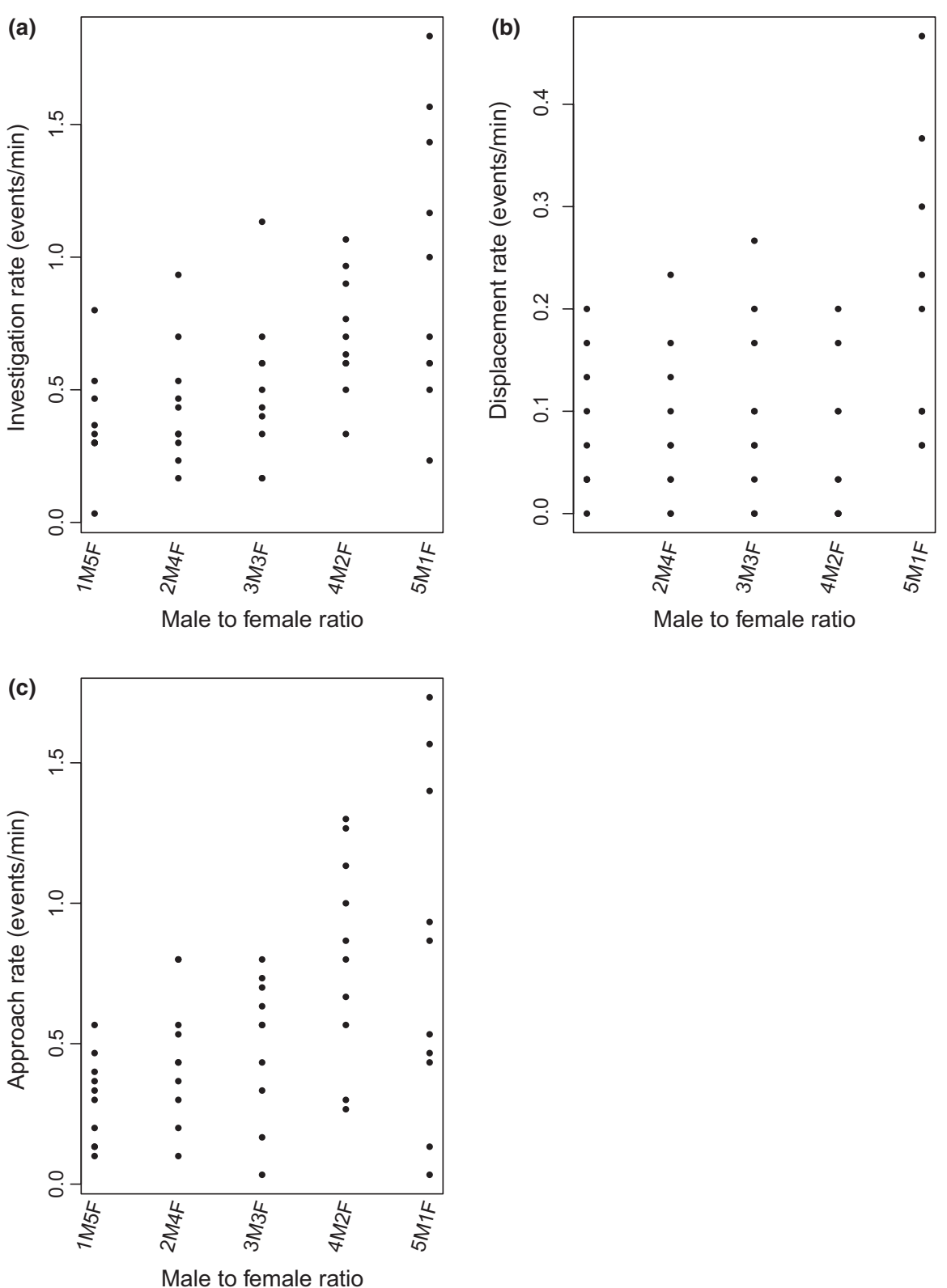

to the core of the network, were significantly higher in females than in males. This is therefore evidence of female social clustering, as females are both more gregarious and more socially integrated within the group's core than are males.

Female social clustering has not previously been demonstrated in cockroach aggregations. Sexual segregation has been found to occur in whirligig beetle (Dineutes discolor) groups following a simulated predator attack, but this structure disappeared after a short time and is likely to be attributed to predator avoidance (Romey \& Wallace, 2007). Large-scale spatial sexual segregation has, however, been demonstrated in a number of insect species where females actively avoid areas with an abundance of males in order to minimise harassment (Parker, 1970, 1978). This explanation could also be used to explain the fine-scale social structure demonstrated here in cockroaches.

Social pressures experienced by females are likely to explain this observed structure since female behaviour appears to drive this network structure, resulting in a core group of females, and a more female-biased sex ratio is likely to be preferable to females. For two of the groups studied, all occurrence behavioural sampling was employed to sample both approach and displacement behaviour carried out and experienced in order to elucidate sex differential social experiences. Neither approach nor displacement networks were found to correlate with the proximity network, meaning there is no evidence either of these behaviours in isolation drives the observed fine-scale social structure. However, we found females carried out the majority of displacements, with males receiving the most displacements, as shown by significantly higher female out-strength centrality and male in-strength centrality in displacement networks; females were also placed consistently higher than males in the dominance hierarchies. From the approach networks, we found females experienced significantly more approaches than males, as demonstrated by their higher in-strength centrality values; there was also 
a trend towards males instigating more approaches. The female social clustering found here is therefore driven by females, who displace males to the periphery of the social group, whilst concurrently showing a level of tolerance towards other females. This clustering behaviour is likely to be an attempt to construct a more favourable social niche (where a focal individual has non-zero inclusive fitness, Saltz et al., 2016). Where sex ratio was manipulated, females were found to experience higher rates of approaches and investigations at more male-biased sex ratios, although there was no association between displacements received and sex ratio, implying females are tolerant towards other females. A more female-biased sex ratio therefore appears to be associated with lower levels of negative social pressure for females.

Given our very limited understanding of the natural history of D. punctata, we use examples from other species to suggest that the avoidance of male harassment is the most plausible explanation for the female social clustering demonstrated here. General female reluctance to mate is extremely prevalent in insects in particular and has therefore been subject to a great deal of research (e.g., Blanckenhorn, Muhlhauser, Morf, Reusch, \& Reuter, 2000; Blyth \& Gilburn, 2011; Hosken, Martin, Born, \& Huber, 2003; Perry, Sharpe, \& Rowe, 2009). As a consequence, females have evolved multiple strategies to minimise male-induced harm (Wigby \& Chapman, 2004). The female social clustering shown here in D. punctata is likely to be explained by such a strategy to cooperatively minimise male harassment, as occurs in a range of species from horses Equus caballus (Linklater et al., 1999) to guppies P. reticulata (Darden \& Croft, 2008). Studies in other species show this can be an effective strategy; more well-connected females were found to have a greater resilience to disturbance by males in the cat shark Scyliorhinus canicula (Jacoby, Busawon, \& Sims, 2010), and female clustering only occurred where male harassment was likely in observations on the Eastern mosquitofish Gambusia holbrooki (Agrillo, Dadda, \& Bisazza, 2006). For D. punctata, a species with no clear breeding season and hence the potential for male harassment throughout the year, it could be particularly beneficial for females to reduce contact from males hence minimising the energetic costs of mating avoidance.

Other potential explanations of female social clustering in $D$. punctata aggregations were considered; these include sex differences in nutritional or thermoregulation requirements, or in competitive ability. Diploptera punctata shows clear sexual dimorphism, with females being significantly larger than males (Marchal et al., 2013). This indicates sex differences in thermoregulatory or nutritional requirements could drive this behaviour. However, since female clustering occurs locally (i.e., within a small aggregation) and during resting, this excludes explanations based upon differential nutritional requirements in this species. In addition, larger females have a lower surface area to volume ratio than males and so would be expected to have a lower relative rate of heat loss (Randall, Burggren, \& French, 1997); this means thermoregulatory advantages that could be gained by grouping together are unlikely to explain female clustering, as this would be more beneficial for males. Scramble competition could provide an explanation if predation risk is higher at the outer edges of an aggregation (Hamilton, 1971); females are certainly superior competitors, as demonstrated here by their higher dominance status, so are able to displace males to the more risky periphery of the group. However, if this were the case, males might be expected to form their own aggregation where they have the opportunity to occupy more central positions; this clearly does not occur in this species, so an explanation based on female social niche construction (and males concurrently remaining in the aggregation for potential access to females) is more plausible. In addition, since females receive significantly more approaches than males, and these approaches involve males touching or pushing at their body and not merely sharing their space, our results indicate male harassment to be the most likely driver of female social clustering. This explanation is supported by later experiments where females' social environments were quantified at differing sex ratios; females experienced significantly higher investigation and approach rates at more male-biased sex ratios. Experiencing repeated approaches by males can in itself be significantly costly to females in terms of a loss in feeding time, increased energy expenditure and an increased risk of predation (Arnqvist \& Nilsson, 2000; Clutton-Brock \& Parker, 1995; Krupa \& Sih, 1993), meaning there is likely to be strong evolutionary pressure favouring behavioural strategies that minimise male harassment. We propose it is therefore clearly advantageous to females to create a female-biased social niche and so reduce the potential costs of male harassment.

Results from this study add to a growing literature base exploring animal sociality and collective behaviour. Here we show that fine-scale social structure can result from social niche construction; females can engineer their social environment to reduce the level of negative social pressures experienced. We therefore provide an example of how individual decisions can result in higher level social structure based on simple sex-based behavioural differences. This study could lead to a number of avenues for further research. Firstly, larger aggregations could be studied, perhaps using automated tracking software to facilitate data collection. In some cockroach species, aggregations can number millions of individuals (Appel \& Rust, 1985); it would therefore be interesting to determine whether the structure found here persists with increased aggregation size. Secondly, this study could be repeated with individuals for which kinship levels are known; since kin-based associations have been previously reported in cockroaches (Lihoreau \& Rivault, 2009), kin-based association choices could lead to additional fine-scale social structure in resting aggregations. Thirdly, to improve our understanding of the evolution of animal sociality, this approach could be used across a range of species to determine other ways by which social niche construction can drive higher level social structure, especially since social niche construction can itself influence evolution (Saltz \& Nuzhdin, 2014). This could also help us to better understand how the collective behaviour of animals results from individual interactions.

To conclude, we show here that fine-scale social structure occurs in D. punctata resting aggregations and can be explained by social niche construction carried out by females. We highlight that social network analysis is a powerful tool for uncovering higher level 
animal social structure, but can also be used to elucidate the behavioural drivers of this structure at an individual level.

\section{ACKNOWLEDGEMENTS}

The authors would like to thank Veronica Cowl, Elspeth Green and Kate Peters for all their help with animal husbandry and the three reviewers for their helpful and constructive comments.

\section{ORCID}

Christina R. Stanley (iD http://orcid.org/0000-0002-5053-4831

\section{REFERENCES}

Agrillo, C., Dadda, M., \& Bisazza, A. (2006). Sexual harassment influences group choice in female mosquitofish. Ethology, 112(6), 592-598. https://doi.org/10.1111/j.1439-0310.2006.01188.x

Altmann, J. (1974). Observational study of behaviour - Sampling methods. Behaviour, 49(3-4), 227-267. https://doi. org/10.1163/156853974X00534

Ame, J. M., Rivault, C., \& Deneubourg, J. L. (2004). Cockroach aggregation based on strain odour recognition. Animal Behaviour, 68, 793801. https://doi.org/10.1016/j.anbehav.2004.01.009

Appel, A. G., \& Rust, M. K. (1985). Outdoor activity and distribution of the smokybrown cockroach, Periplaneta fuliginosa (Dictyoptera, Blattidae). Environmental Entomology, 14(6), 669-673. https://doi. org/10.1093/ee/14.6.669

Arnqvist, G., \& Nilsson, T. (2000). The evolution of polyandry: Multiple mating and female fitness in insects. Animal Behaviour, 60, 145-164. https://doi.org/10.1006/anbe.2000.1446

Bell, W. J., Roth, L. M., \& Nalepa, C. A. (2007). Cockroaches: Ecology, behaviour and natural history. Baltimore, MD: John Hopkins University Press.

Bergmüller, R., \& Taborsky, M. (2010). Animal personality due to social niche specialisation. Trends in Ecology \& Evolution, 25(9), 504-511. https://doi.org/10.1016/j.tree.2010.06.012

Blanckenhorn, W. U., Muhlhauser, C., Morf, C., Reusch, T., \& Reuter, M. (2000). Female choice, female reluctance to mate and sexual selection on body size in the dung fly Sepsis cynipsea. Ethology, 106(7), 577-593. https://doi.org/10.1046/j.1439-0310.2000.00573.x

Blyth, J. E., \& Gilburn, A. S. (2011). The function of female behaviours adopted during premating struggles in the seaweed fly, Coelopa frigida. Animal Behaviour, 81(1), 77-82. https://doi.org/10.1016/j. anbehav.2010.09.013

Borgatti, S. P. (2002). NetDraw: Graph visualization software. Harvard, MA: Analytic Technologies.

Borgatti, S. P., \& Everett, M. G. (1999). Models of core/periphery structures. Social Networks, 21(4), 375-395.

Borgatti, S. P., Everett, M. G., \& Freeman, L. C. (2002). UCINET for windows: Software for social network analysis. Harvard, MA: Analytic Technologies.

Borgatti, S., Everett, M., \& Freeman, L. (2006). UCINET for windows: Software for social network analysis. Cambridge, MA: Analytic Technologies.

Brask, J. B., Croft, D. P., Thompson, K., Dabelsteen, T., \& Darden, S. K. (2012). Social preferences based on sexual attractiveness: A female strategy to reduce male sexual attention. Proceedings of the Royal Society B-Biological Sciences, 279(1734), 1748-1753. https://doi. org/10.1098/rspb.2011.2212
Cairns, S. J., \& Schwager, S. J. (1987). A comparison of association indices. Animal Behaviour, 35(5), 1454-1469. https://doi.org/10.1016/ S0003-3472(87)80018-0

Carrington, P. J., Scott, J. \& Wasserman, S. 2005. Models and methods in social network analysis. Cambridge, UK: Cambridge University Press. https://doi.org/10.1017/СBO9780511811395

Charbonneau, D., Blonder, B. \& Dornhaus, A. 2013. Social insects: A model system for network dynamics. In P. Holme \& J. Saramaki (Eds.), Temporal networks, understanding complex systems (pp. 217244). Berlin, Germany: Springer-Verlag.

Clutton-Brock, T. H., \& Parker, G. A. (1995). Sexual coercion in animal societies. Animal Behaviour, 49(5), 1345-1365. https://doi. org/10.1006/anbe.1995.0166

Costa, J. T. (2006). The other insect societies. Cambridge, MA: The Belknap Press of Harvard University Press.

Crissman, J. R., Booth, W., Santangelo, R. G., Mukha, D. V., Vargo, E. L., \& Schal, C. (2010). Population genetic structure of the German Cockroach (Blattodea: Blattellidae) in apartment buildings. Journal of Medical Entomology, 47(4), 553-564. https://doi.org/10.1093/ jmedent/47.4.553

Croft, D. B., James, R., \& Krause, J. (2008). Exploring animal social networks. Princeton, NJ: Princeton University Press. https://doi. org/10.1515/9781400837762

Daly, M. (1978). Cost of mating. American Naturalist, 112(986), 771-774. https://doi.org/10.1086/283319

Darden, S. K., \& Croft, D. P. (2008). Male harassment drives females to alter habitat use and leads to segregation of the sexes. Biology letters, 4(5), 449-451.

David, H. A. (1988). The method of paired comparisons. London, UK: C. Griffin.

Everett, M. \& Borgatti, S. 2005. Extending centrality. In P. J. Carrington, J. Scott \& S. Wasserman (Eds.), Models and methods in social network analysis (pp. 57-76). Cambridge, UK: Cambridge University Press. https://doi.org/10.1017/СBO9780511811395

Fewell, J. H. (2003). Social insect networks. Science, 301(5641), 18671870. https://doi.org/10.1126/science.1088945

Fisher, D. N., Rodríguez-Muñoz, R., \& Tregenza, T. (2016a). Comparing pre-and post-copulatory mate competition using social network analysis in wild crickets. Behavioral Ecology, 27(3), 912-919. https:// doi.org/10.1093/beheco/arv236

Fisher, D. N., Rodríguez-Muñoz, R., \& Tregenza, T. (2016b). Wild cricket social networks show stability across generations. BMC Evolutionary Biology, 16(1), 151. https://doi.org/10.1186/s12862-016-0726-9

Formica, V. A., Wood, C. W., Larsen, W. B., Butterfield, R. E., Augat, M. E., Hougen, H. Y., \& Brodie, E. D. (2012). Fitness consequences of social network position in a wild population of forked fungus beetles (Bolitotherus cornutus). Journal of Evolutionary Biology, 25(1), 130-137. https://doi.org/10.1111/j.1420-9101.2011.02411.x

Fu, F., Nowak, M. A., Christakis, N. A., \& Fowler, J. H. (2012). The evolution of homophily. Scientific Reports, 2, 845. https://doi.org/10.1038/ srep00845

Fukui, M., \& Takahashi, S. (1980). Studies on the mating behaviour of the cockroach Nauphoeta cinerea olivier. 1. Sex discrimination by males. Applied Entomology and Zoology, 15(1), 20-26. https://doi. org/10.1303/aez.15.20

Hamilton, W. D. (1971). Geometry for the selfish herd. Journal of Theoretical Biology, 31(2), 295-311. https://doi. org/10.1016/0022-5193(71)90189-5

Hanneman, R. A., \& Riddle, M. (2005). Introduction to social network methods. Riverside, CA: University of California, Riverside.

Holbrook, G. L., \& Schal, C. (1998). Social influences on nymphal development in the cockroach, Diploptera punctata. Physiological Entomology, 23(2), 121-130. https://doi. org/10.1046/j.1365-3032.1998.232077.x 
Holbrook, G. L., \& Schal, C. (2004). Maternal investment affects offspring phenotypic plasticity in a viviparous cockroach. Proceedings of the National Academy of Sciences of the United States of America, 101(15), 5595-5597. https://doi.org/10.1073/pnas.0400209101

Hosken, D. J., Martin, O. Y., Born, J., \& Huber, F. (2003). Sexual conflict in Sepsis cynipsea: Female reluctance, fertility and mate choice. Journal of Evolutionary Biology, 16(3), 485-490. https://doi. org/10.1046/j.1420-9101.2003.00537.x

Inghilesi, A. F., Mazza, G., Cervo, R., \& Cini, A. (2015). A network of sex and competition: The promiscuous mating system of an invasive weevil. Current Zoology, 61(1), 85-97. https://doi.org/10.1093/ czoolo/61.1.85

Jacoby, D. M. P., Busawon, D. S., \& Sims, D. W. (2010). Sex and social networking: The influence of male presence on social structure of female shark groups. Behavioral Ecology, 21(4), 808-818. https:// doi.org/10.1093/beheco/arq061

Jeanson, R. (2012). Long-term dynamics in proximity networks in ants. Animal Behaviour, 83(4), 915-923. https://doi.org/10.1016/j. anbehav.2012.01.009

Jeanson, R., Rivault, C., Deneubourg, J. L., Blanco, S., Fournier, R., Jost, C., \& Theraulaz, G. (2005). Self-organized aggregation in cockroaches. Animal Behaviour, 69, 169-180. https://doi.org/10.1016/j. anbehav.2004.02.009

Kohn, G. M., King, A. P., Scherschel, L. L., \& West, M. J. (2011). Social niches and sex assortment: Uncovering the developmental ecology of brown-headed cowbirds, Molothrus ater. Animal Behaviour, 82(5), 1015-1022. https://doi.org/10.1016/j.anbehav.2011.07.035

Krackhardt, D. (1988). Predicting with networks - Nonparametric multiple-regression analysis of dyadic data. Social Networks, 10(4), 359381. https://doi.org/10.1016/0378-8733(88)90004-4

Kralj-Fiser, S., \& Schuett, W. (2014). Studying personality variation in invertebrates: Why bother? Animal Behaviour, 91, 41-52. https://doi. org/10.1016/j.anbehav.2014.02.016

Krause, J., Croft, D. P., \& James, R. (2007). Social network theory in the behavioural sciences: Potential applications. Behavioral Ecology and Sociobiology, 62(1), 15-27. https://doi.org/10.1007/ s00265-007-0445-8

Krupa, J. J., \& Sih, A. (1993). Experimental studies on water strider mating dynamics: Spatial variation in density and sex ratio. Behavioral Ecology and Sociobiology, 33(2), 107-120.

Kurvers, R., Krause, J., Croft, D. P., Wilson, A. D. M., \& Wolf, M. (2014). The evolutionary and ecological consequences of animal social networks: Emerging issues. Trends in Ecology \& Evolution, 29(6), 326335. https://doi.org/10.1016/j.tree.2014.04.002

Lange, R., Reinhardt, K., Michiels, N. K., \& Anthes, N. (2013). Functions, diversity, and evolution of traumatic mating. Biological Reviews, 88(3), 585-601. https://doi.org/10.1111/brv.12018

Legendre, P., \& Fortin, M. J. (2010). Comparison of the Mantel test and alternative approaches for detecting complex multivariate relationships in the spatial analysis of genetic data. Molecular Ecology Resources, 10(5), 831-844. https://doi. org/10.1111/j.1755-0998.2010.02866.x

Lihoreau, M., Costa, J. T., \& Rivault, C. (2012). The social biology of domiciliary cockroaches: Colony structure, kin recognition and collective decisions. Insectes Sociaux, 59(4), 445-452. https://doi. org/10.1007/s00040-012-0234-x

Lihoreau, M., \& Rivault, C. (2009). Kin recognition via cuticular hydrocarbons shapes cockroach social life. Behavioral Ecology, 20(1), 46-53. https://doi.org/10.1093/beheco/arn113

Linklater, W. L., Cameron, E. Z., Minot, E. O., \& Stafford, K. J. (1999). Stallion harassment and the mating system of horses. Animal Behaviour, 58, 295-306. https://doi.org/10.1006/anbe.1999.1155

Lusseau, D., \& Newman, M. E. J. (2004). Identifying the role that animals play in their social networks. Proceedings Royal Society of London Series B, 271, S477-S481. https://doi.org/10.1098/rsbl.2004.0225
Marchal, E., Hult, E. F., Huang, J., Stay, B., \& Tobe, S. S. (2013) Diploptera punctata as a model for studying the endocrinology of arthropod reproduction and development. General and Comparative Endocrinology, 188, 85-93. https://doi.org/10.1016/j. ygcen.2013.04.018

Muniz, D. G., Guimarães, P. R. Jr, Buzatto, B. A., \& Machado, G. (2014). A sexual network approach to sperm competition in a species with alternative mating tactics. Behavioral Ecology, 26(1), 121-129.

Naug, D. (2009). Structure and resilience of the social network in an insect colony as a function of colony size. Behavioral Ecology and Sociobiology, 63(7), 1023-1028. https://doi.org/10.1007/ s00265-009-0721-x

Opsahl, T. (2009). Structure and evolution of weighted networks, PhD. London, UK: University of London, Queen Mary College.

Opsahl, T., \& Panzarasa, P. (2009). Clustering in weighted networks. Social Networks, 31(2), 155-163. https://doi.org/10.1016/j. socnet.2009.02.002

Parker, G. A. (1970). The reproductive behaviour and the nature of sexual selection in Scatophaga stercoraria L.V. The female's behaviour at the oviposition site. Behaviour, 37, 140-168. https://doi. org/10.1163/156853970X00277

Parker, G. A. (1978). The evolution of competitive mate searching. Annual Review of Entomology, 23, 173-196. https://doi.org/10.1146/annurev.en.23.010178.001133

Parrish, J. K., \& Edelstein-Keshet, L. (1999). Complexity, pattern, and evolutionary trade-offs in animal aggregation. Science, 284(5411), 99-101. https://doi.org/10.1126/science.284.5411.99

Perry, J. C., Sharpe, D. M. T., \& Rowe, L. (2009). Condition-dependent female remating resistance generates sexual selection on male size in a ladybird beetle. Animal Behaviour, 77(3), 743-748. https://doi. org/10.1016/j.anbehav.2008.12.013

Pilastro, A., Benetton, S., \& Bisazza, A. (2003). Female aggregation and male competition reduce costs of sexual harassment in the mosquitofish Gambusia holbrooki. Animal Behaviour, 65, 1161-1167. https:// doi.org/10.1006/anbe.2003.2118

R Development Core Team (2013). R: A language and environment for statistical computing. Vienna, Austria: R Foundation for Statistical Computing.

Randall, E., Burggren, W., \& French, K. (1997). Animal physiology. New York, NY: W H Freeman and Company.

Reale, D., Bousses, P., \& Chapuis, J. L. (1996). Female-biased mortality induced by male sexual harassment in a feral sheep population. Canadian Journal of Zoology-Revue Canadienne De Zoologie, 74(10), 1812-1818. https://doi.org/10.1139/z96-202

Romey, W. L., \& Wallace, A. C. (2007). Sex and the selfish herd: Sexual segregation within nonmating whirligig groups. Behavioral Ecology, 18(5), 910-915. https://doi.org/10.1093/beheco/arm057

Rowe, L. (1994). The costs of mating and mate choice in water striders. Animal Behaviour, 48(5), 1049-1056. https://doi.org/10.1006/ anbe.1994.1338

Saltz, J. B., \& Foley, B. R. (2011). Natural genetic variation in social niche construction: Social effects of aggression drive disruptive sexual selection in Drosophila melanogaster. The American Naturalist, 177(5), 645-654. https://doi.org/10.1086/659631

Saltz, J. B., Geiger, A. P., Anderson, R., Johnson, B., \& Marren, R. (2016). What, if anything, is a social niche? Evolutionary Ecology, 30(2), 349364. https://doi.org/10.1007/s10682-015-9792-5

Saltz, J. B., \& Nuzhdin, S. V. (2014). Genetic variation in niche construction: Implications for development and evolutionary genetics. Trends in Ecology \& Evolution, 29(1), 8-14. https://doi.org/10.1016/j. tree.2013.09.011

Seelinger, G., \& Schuderer, B. (1985). Release of male courtship display in Periplaneta americana - Evidence for female contact sex pheromone. Animal Behaviour, 33, 599-607. https://doi.org/10.1016/ S0003-3472(85)80084-1 
Sempo, G., Canonge, S., Detrain, C., \& Deneubourg, J.-L. (2009). Complex dynamics based on a quorum: Decision-making process by cockroaches in a patchy environment. Ethology, 115(12), 1150-1161. https://doi.org/10.1111/j.1439-0310.2009.01699.x

Sih, A., Hanser, S. F., \& McHugh, K. A. (2009). Social network theory: New insights and issues for behavioral ecologists. Behavioral Ecology and Sociobiology, 63(7), 975-988. https://doi.org/10.1007/ s00265-009-0725-6

Silk, J. B. (2007). Social components of fitness in primate groups. Science, 317(5843), 1347-1351. https://doi.org/10.1126/science.1140734

Smuts, B. B. \& Smuts, R. W. 1993. Male aggression and sexual coercion of females in nonhuman primates and other mammals - Evidence and theoretical implications. In P. J. B. Slater, J. S. Rosenblatt, C. T. Snowdon \& M. Milinski (Eds.), Advances in the study of behavior (Vol. 22, pp. 1-63). Elsevier Academic Press Inc., San Diego, CA.

Stanley, C. R., Mettke-Hofmann, C., \& Preziosi, R. F. (2017). Personality in the cockroach Diploptera punctata: Evidence for stability across developmental stages despite age effects on boldness. PLoSOne,12(5), e0176564.https://doi.org/10.1371/journal.pone. 0176564

Sumpter, D. J. (2006). The principles of collective animal behaviour. Philosophical Transactions of the Royal Society B: Biological Sciences, 361(1465), 5-22. https://doi.org/10.1098/rstb.2005.1733

Sundaresan, S. R., Fischhoff, I. R., Dushoff, J., \& Rubenstein, D. I. (2007). Network metrics reveal differences in social organization between two fission-fusion species, Grevys zebra and onager. Oecologia, 151, 140-149. https://doi.org/10.1007/s00442-006-0553-6
Wasserman, S., \& Faust, K. (1994). Social network analysis - Methods and applications. Cambridge, MA: Cambridge University Press. https:// doi.org/10.1017/CBO9780511815478

Wey, T., Blumstein, D. T., Shen, W., \& Jordan, F. (2008). Social network analysis of animal behaviour: A promising tool for the study of sociality. Animal Behaviour, 75, 333-344. https://doi.org/10.1016/j. anbehav.2007.06.020

Whitehead, H. (2009). SOCPROG programs: Analysing animal social structures. Behavioral Ecology and Sociobiology, 63(5), 765-778. https://doi.org/10.1007/s00265-008-0697-y

Wigby, S., \& Chapman, T. (2004). Female resistance to male harm evolves in response to manipulation of sexual conflict. Evolution, 58(5), 1028-1037. https://doi.org/10.1111/j.0014-3820.2004.tb00436.x

Wilson, W. G., \& Richards, S. A. (2000). Consuming and grouping: Resource-mediated animal aggregation. Ecology Letters, 3(3), 175180. https://doi.org/10.1046/j.1461-0248.2000.00135.x

Woodhead, A. P. (1985). Sperm mixing in the cockroach Diploptera punctata. Evolution, 39(1), 159-164. https://doi. org/10.1111/j.1558-5646.1985.tb04088.x

How to cite this article: Stanley CR, Liddiard Williams $\mathrm{H}$, Preziosi RF. Female clustering in cockroach aggregations-A case of social niche construction? Ethology. 2018;124:706718. https://doi.org/10.1111/eth.12799 